\title{
Atividade reprodutiva do peixe-espada, Trichiurus lepturus (Teleostei, Trichiuridae), vulnerável à pesca de pequena escala no extremo-norte do litoral de Santa Catarina, Brasil
}

\author{
Soraya Vitório Del Puente \\ Paulo de Tarso Chaves* \\ Departamento de Zoologia, Universidade Federal do Paraná \\ Caixa Postal 19020, CEP 81531-980, Curitiba - PR, Brasil \\ *Autor para correspondência \\ ptchaves@ufpr.br
}

\section{Resumo}

O peixe-espada, Trichiurus lepturus, foi investigado nos desembarques da pesca de pequena escala realizada no litoral norte de Santa Catarina, objetivando avaliar época e artes em que a pesca incide sobre indivíduos em reprodução. Analisaram-se 398 exemplares, amostrados entre agosto de 2006 e agosto de 2007, provenientes das três artes mais praticadas na região: fundeio, caceio e arrasto. Fêmeas são maiores que machos, o que torna as redes de emalhe mais seletivas para aquele sexo que o arrasto.

Estimou-se que fêmeas maturam pela primeira vez com $75,0 \mathrm{~cm}$, em média, enquanto machos com $64,0 \mathrm{~cm}$. Fundeio, caceio e arrasto são eficientes para indivíduos jovens e adultos, todavia indivíduos em reprodução foram registrados apenas nos dois primeiros. Ocorrência de fêmeas em hidratação pré-ovulatória e pós-desova, de machos maduros, e os valores elevados de Índice Gonadossomático (IGS), indicam que as pescas de fundeio e caceio atuam sobre indivíduos em atividade reprodutiva ao longo de todo o ano, com picos na primavera e no verão. Visto que no litoral norte de Santa Catarina o arrasto camaroeiro é interditado na primavera, recomenda-se reduzir a intensidade de uso de redes de emalhe durante a primavera e principalmente durante o verão, aliviando assim a pressão por pesca de indivíduos reprodutivos.

Unitermos: reprodução, primeira maturação, desova, peixe-espada, sul do Brasil

\section{Abstract}

Reproductive status of the swordfish, Trichiurus lepturus (Teleostei, Trichiuridae), exposed to small-scale fishing on the north coast of Santa Catarina state, southern Brazil. The swordfish, Trichiurus lepturus, caught by small-scale fishermen from a community of southern Brazil was investigated based on 398 individuals landed from August 2006 to August 2007, aiming to evaluate in which periods and fishing techniques the reproductive individuals of this species are most exposed to fishing activities. The analysis took into consideration the length of individuals, the morphological attributes of their gonads, the Gonadossomatic 
Index (GSI), and the kind of gear used in the catches. It was estimated that females mature for the first time at a total length of $75.0 \mathrm{~cm}$, and males at $64.0 \mathrm{~cm}$. Gillnets and bottom trawling were efficient for the capture of both young and adults, but reproductive individuals were registered only in gillnets. Oocyte hydration and postspawning in females, the occurrence of mature gonads in males and the values of GSI indicate that "fundeio" and "caceio" (different types of gillnets) are acting on reproductive individuals throughout the entire year, with maximum intensity in spring and summer. Considering that in the studied region fisheries are closed for shrimp bottom trawling in spring, it is suggested that the use of gillnets during spring and mainly summer should be reduced, thus improving the spawning process.

Key words: breeding, first maturation, spawning, swordfish, southern Brazil

\section{Introdução}

A atividade pesqueira no litoral de Santa Catarina destaca-se não apenas por seus indicadores na modalidade industrial, mas também por aquela dita artesanal, ou de pequena escala (Sunyé, 2006). Registros da EPAGRISC (2004, apud Sunyé, 2006) contabilizam em 2003 aproximadamente 38.000 pessoas e 6.000 embarcações atuando em 75 pontos de desembarque. No norte do Estado a atividade baseia-se nas artes fundeio, caceio e arrasto, as duas primeiras utilizando redes de emalhe e a última, redes de arrasto de fundo com portas ou pranchas (Robert e Chaves, 2006). O arrasto visa à captura do camarão sete-barbas, Xiphopenaeus kroyeri Heller, 1862, mas as três artes são eficazes na captura de peixes.

O peixe-espada, Trichiurus lepturus Linnaeus, 1758, é de ocorrência comum em todas (Chaves e Robert, 2003; Souza e Chaves, 2007). Entre 1988 e 1997 ele ocupou a quarta colocação em captura dentre as espécies de maior importância na pesca catarinense de pequena escala, e vem assumindo importância crescente na estatística pesqueira do Sudeste-Sul do Brasil (Sunyé, 2006). Segundo Magro (2005), na região Sudeste o consumo dessa espécie tem aumentado, e em Santa Catarina a exportação já é realidade.

Atributos biológicos de Trichiurus lepturus são descritos em populações do Brasil (Martins e Haimovici, 2000; Magro, 2006), da China (Yamada, 1970; Park et al., 1998;KWok e Ni, 1999), do Japão (Munekiyo e Kuwahara, 1984a e b) e de Taiwan (Jean e Lee, 1984). Trata-se de uma espécie com hábito demerso-pelágico e que a partir de $50 \mathrm{~cm}$ compõe cardumes migrantes, que também se deslocam verticalmente (Magro, 2005). Em conseqüência, indivíduos da espécie são vulneráveis tanto às artes de pesca de superfície como àquelas de fundo. O presente trabalho analisa a condição reprodutiva dos indivíduos que vêm sendo capturados pela pesca de pequena escala praticada no extremo norte do litoral de Santa Catarina. Objetiva-se avaliar em que época e com que tipo de arte a frota de pequena escala está atuando em maior intensidade sobre a parcela reprodutiva da população em maior intensidade.

\section{Material e Métodos}

Amostragens de T. lepturus foram realizadas mensalmente entre agosto de 2006 e agosto de 2007 nos desembarques da comunidade de Barra do Saí (25⒌'S; $\left.48^{\circ} 36^{\prime} \mathrm{W}\right)$, município de Itapoá, litoral norte de Santa Catarina, totalizando 398 exemplares provenientes de fundeio, caceio e arrasto (Tabela 1). De cada exemplar foram registrados o comprimento total (CT) e o peso (PT). O sexo e o estádio de maturação foram diagnosticados por análise das gônadas. Estas foram pesadas (PG) e, em parte, fixadas em formol $4 \%$ para confecção de preparados histológicos permanentes, corados com HematoxilinaEosina (HE) (Vazzoler, 1996). A proporção sexual foi testada pelo teste de Qui-quadrado $\left(\chi^{2}\right)$, com 1 grau de liberdade e $5 \%$ de significância $\left(\chi^{2}>3,84\right)$. A condição reprodutiva foi analisada mensalmente e por classe de comprimento com auxílio da seguinte escala de estádios de maturação gonadal, baseada em Vazzoler (1996): em ambos os sexos, A - imaturo; B - em maturação; $\mathrm{C}$ - maduro; apenas em fêmeas, $\mathrm{Ch}$ - em hidratação (desova iminente) e D - semi-desovado, desovado ou em recuperação; e apenas em machos, $\mathrm{E}$ - semi-espermiado, espermiado ou em recuperação.

A freqüência de ocorrência dos estádios de maturação foi determinada nos meses em que o número de exemplares de um mesmo sexo foi igual ou superior 
a cinco. Assumiu-se a ocorrência dos estádios C, $\mathrm{Ch}, \mathrm{D}$ e E como indicadora de atividade reprodutiva. Em caráter acessório, para estimativa da atividade reprodutiva calculou-se individualmente o valor de Índice Gonadossomático (IGS), definido como IGS = (PG/PT) x 100. Para análise sazonal os meses foram agrupados por: inverno (de agosto a setembro de 2006 e julho e agosto de 2007); primavera (de outubro a dezembro de 2006); verão (de janeiro a março de 2007); e outono (de abril a junho de 2007).

O comprimento médio de primeira maturação foi estimado com auxílio do programa estatístico Origin 50 (Jocic-Jakubi e Jovic, 2006), que fornece valores da variável independente $(\mathrm{CT})$ a partir daqueles da variável dependente (freqüência de indivíduos adultos $-\mathrm{B}, \mathrm{C}$, $\mathrm{Ch}, \mathrm{D}$ ou E) numa distribuição cuja curva tem tendência sigmóide.

\section{Resultados}

Os exemplares de $T$. lepturus registrados nos desembarques mediam entre 19,0 e $145,8 \mathrm{~cm}$, situando-se $63 \%$ deles entre 84,0 e $110,0 \mathrm{~cm}$. O maior macho e a maior fêmea mediam $120,0 \mathrm{~cm}$ e $145,8 \mathrm{~cm}$, respectivamente. $\mathrm{O}$ arrasto foi responsável pela captura dos exemplares de porte menor, enquanto o caceio e o fundeio pela dos de porte maior (Tabela 1).
A proporção sexual foi significativamente diferente de 1:1 $\left(\chi^{2}>3,84\right)$ em todos os meses, exceto dezembro e janeiro. Em novembro houve predomínio de machos, enquanto que nos demais meses houve predomínio de fêmeas. No período como um todo a proporção entre machos e fêmeas foi 1:2. Proporções significativamente diferentes de 1:1 ocorreram também em todas as classes de comprimento, exceto em $32-45 \mathrm{~cm}$ e $84-97 \mathrm{~cm}$. Nos tamanhos maiores, a partir de $97,0 \mathrm{~cm}$ predominaram fêmeas, sendo que a partir de $123,0 \mathrm{~cm}$ apenas esse sexo foi registrado. Exemplares com sexo indeterminado ocorreram somente nas três classes de tamanho menores, compondo-se a primeira delas integralmente por exemplares nessa condição.

Fêmeas em maturação foram registradas com freqüência superior a $10 \%$ desde a primavera até meados do inverno (Figura 1). Após isso, a partir do final do inverno (setembro de 2006), fêmeas maduras ocorreram com freqüência superior a $10 \%$ até meados do outono (abril de 2007); em parte desse período - de novembro de 2006 a fevereiro de 2007 - ocorreram fêmeas em hidratação pré-ovulatória (Figura 2). Fêmeas desovadas foram registradas em parte da primavera $\mathrm{e}$ no verão, bem como no inverno (Figuras 1 e 2). A frota que desembarca na região estudada incidiu também sobre machos maduros, em todas as estações do ano. Entretanto, exemplares espermiados foram registrados apenas no inverno de 2007 (Figura 3).

TABELA 1: Número de exemplares de Trichiurus lepturus analisados (n) segundo a arte de pesca e o mês (agosto de 2006 a agosto de 2007), e valores de comprimento total mínimo e máximo (CT).

\begin{tabular}{|c|c|c|c|c|c|c|c|}
\hline \multirow{2}{*}{ Meses } & \multicolumn{2}{|c|}{ Fundeio } & \multicolumn{2}{|c|}{ Caceio } & \multicolumn{2}{|c|}{ Arrasto } & \multirow{2}{*}{ Total } \\
\hline & $\mathbf{n}$ & CT (cm) & $\mathrm{n}$ & CT (cm) & $\mathrm{n}$ & CT $(\mathrm{cm})$ & \\
\hline Ago./06 & 5 & $71,0-79,0$ & - & - & - & - & 5 \\
\hline Set./06 & 28 & $80,1-131,0$ & - & - & - & - & 28 \\
\hline Out./06 & 8 & $73,2-109,8$ & 9 & $88,7-110,6$ & - & - & 17 \\
\hline Nov./06 & 52 & $83,0-109,0$ & - & - & - & - & 52 \\
\hline Dez./06 & 37 & $72,1-120,0$ & - & - & 52 & $19,0-57,3$ & 89 \\
\hline Jan./07 & 6 & $84,0-101,0$ & 3 & $96,1-97,1$ & - & - & 9 \\
\hline Fev./07 & 31 & $66,9-109,0$ & - & - & - & - & 31 \\
\hline Mar./07 & 21 & $80,6-113,0$ & 4 & $70,3-108,1$ & 25 & $31,8-51,0$ & 50 \\
\hline Abr./07 & 23 & $82,4-106,8$ & - & - & 3 & $69,0-88,3$ & 26 \\
\hline Maio/07 & - & - & 1 & 113,5 & - & - & 1 \\
\hline Jun./07 & 9 & $95,3-104,1$ & 12 & $69,9-123,8$ & 5 & $42,8-56,5$ & 26 \\
\hline Jul./07 & 41 & $88,3-145,8$ & 5 & $85,3-115,3$ & - & - & 46 \\
\hline Ago./07 & 11 & $85,4-126,2$ & 7 & $79,4-104,4$ & - & - & 18 \\
\hline TOTAL & 272 & $71,0-145,8$ & 41 & $69,9-123,8$ & 85 & $19,0-88,3$ & 398 \\
\hline
\end{tabular}




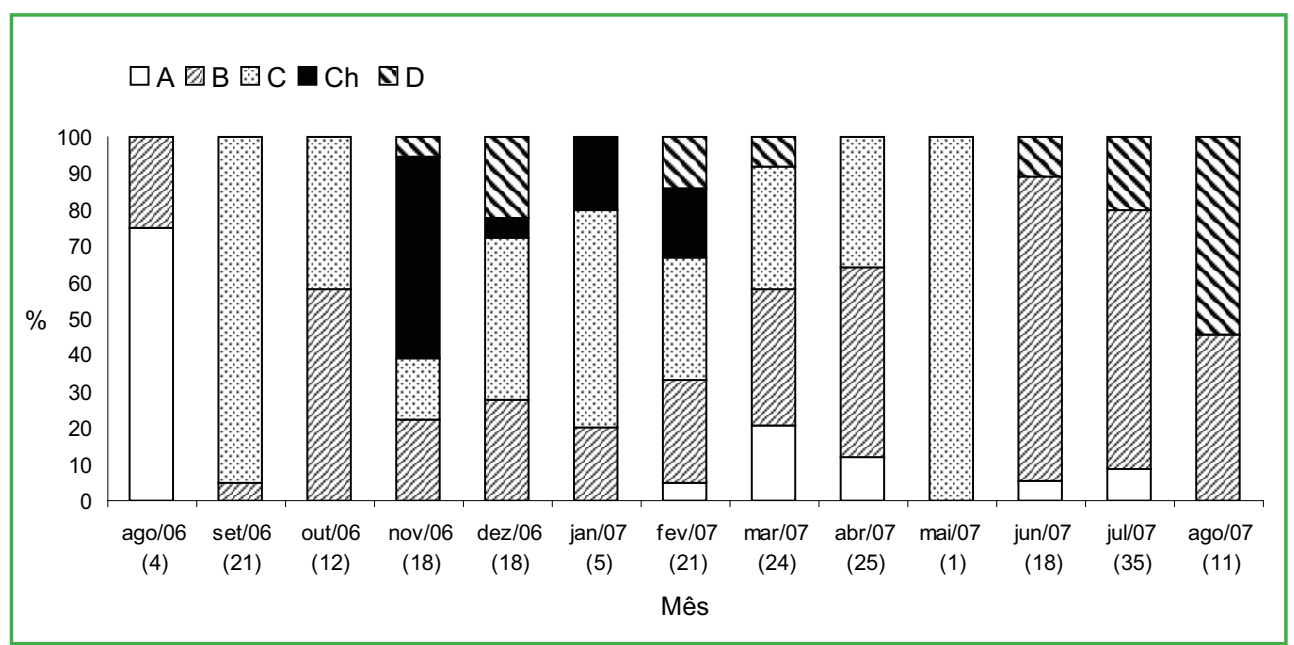

FIGURA 1: Freqüência mensal de ocorrência dos estádios de maturação em fêmeas de Trichiurus lepturus desembarcadas entre agosto de 2006 e agosto de 2007. Entre parênteses, o tamanho da amostra. A: imaturo; B: em maturação; C: maduro; Ch: em hidratação; D: semi-desovado, desovado ou em recuperação.

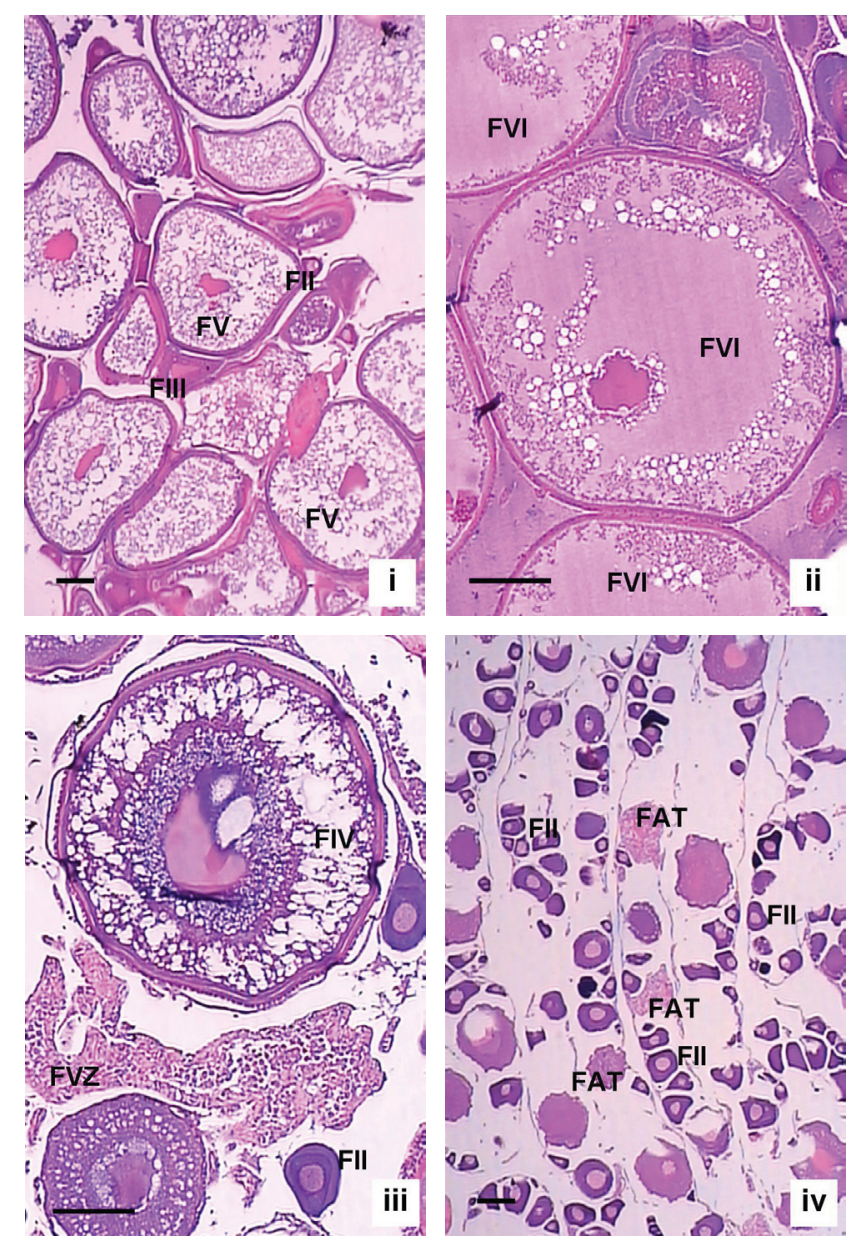

FIGURA 2: Cortes histológicos de ovários de Trichiurus lepturus. i) Maduro, estádio C. 10X4. ii) Em hidratação, estádio Ch. $10 X 10$. iii) Desovado, estádio D. 10X10. iv) Em recuperação, estádio D. 10X4. Folículos ovarianos: FII: pré-vitelogênicos; FIII: em vitelogênese inicial; FIV: em vitelogênese avançada; FV: em vitelogênese completa; FVI: hidratado; FVZ: vazio, pós-ovulatório; FAT: atrésicos. HE. Barra $=100 \mathrm{~mm}$. 


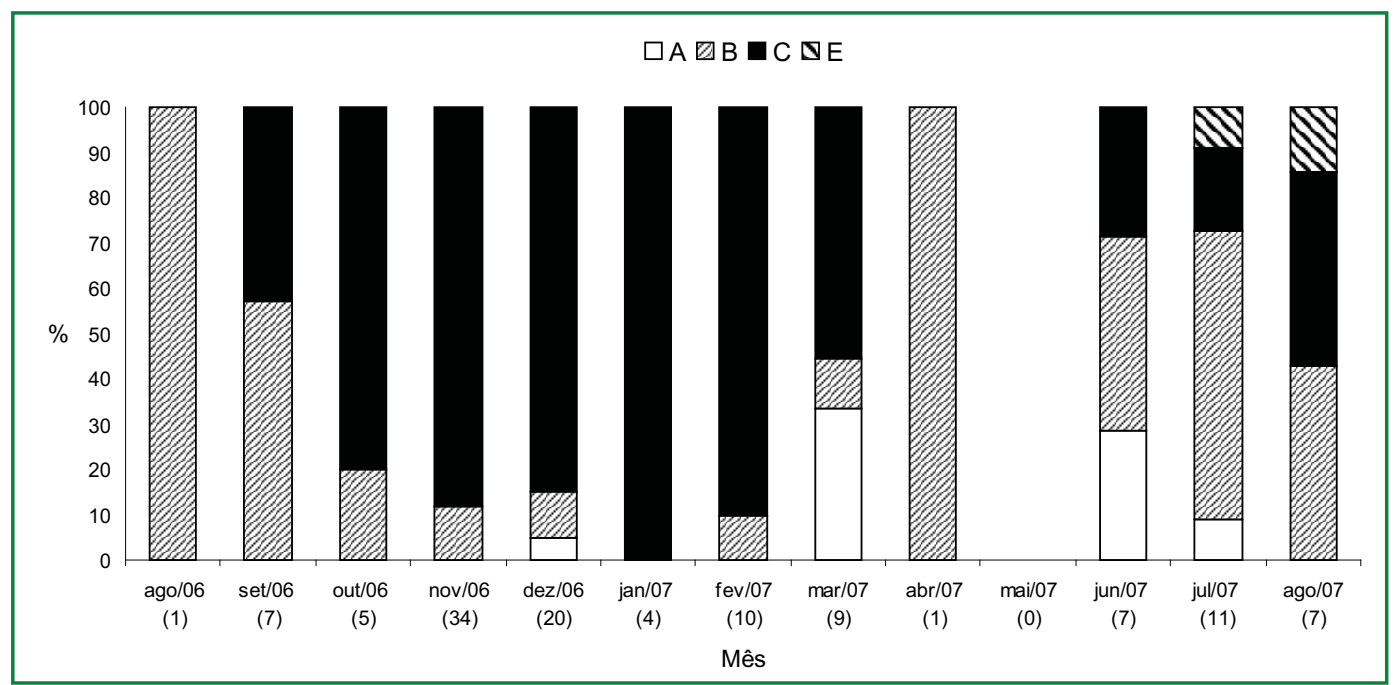

FIGURA 3: Freqüência mensal de ocorrência dos estádios de maturação em machos de Trichiurus lepturus desembarcados entre agosto de 2006 e agosto de 2007. Entre parênteses, o tamanho da amostra. A: imaturo; B: em maturação; C: maduro; E: semi-espermiado, espermiado ou em recuperação.

Nas fêmeas os maiores valores individuais de IGS foram constatados em novembro, dezembro e fevereiro (Figura 4), e nos machos em novembro e março
(Figura 5). Nas fêmeas o comprimento médio de primeira maturação foi estimado em $75,0 \mathrm{~cm}$; nos machos, em $64,0 \mathrm{~cm}$.

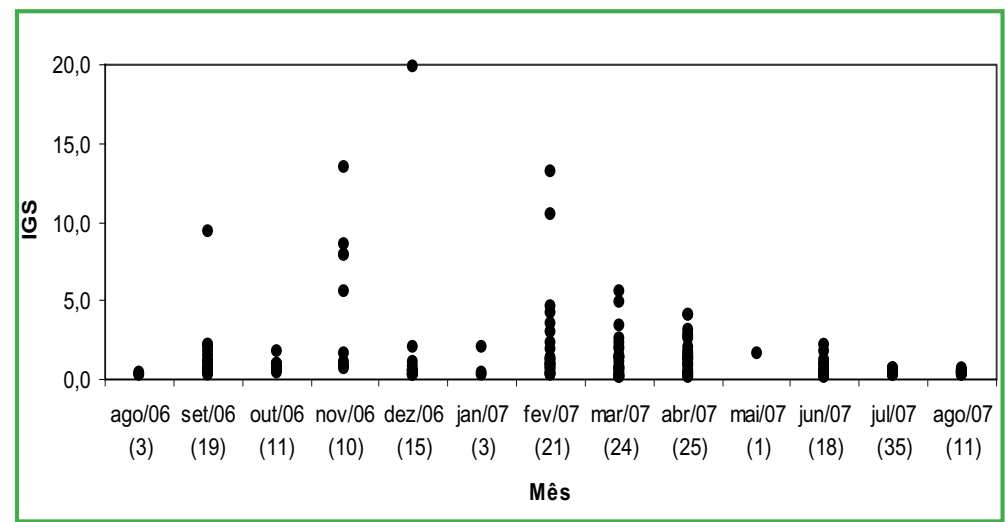

FIGURA 4: Distribuição mensal dos valores individuais de IGS de fêmeas de Trichiurus lepturus desembarcadas pela pesca de pequena escala no litoral norte de Santa Catarina, entre agosto de 2006 e agosto de 2007. Entre parênteses, o tamanho da amostra.

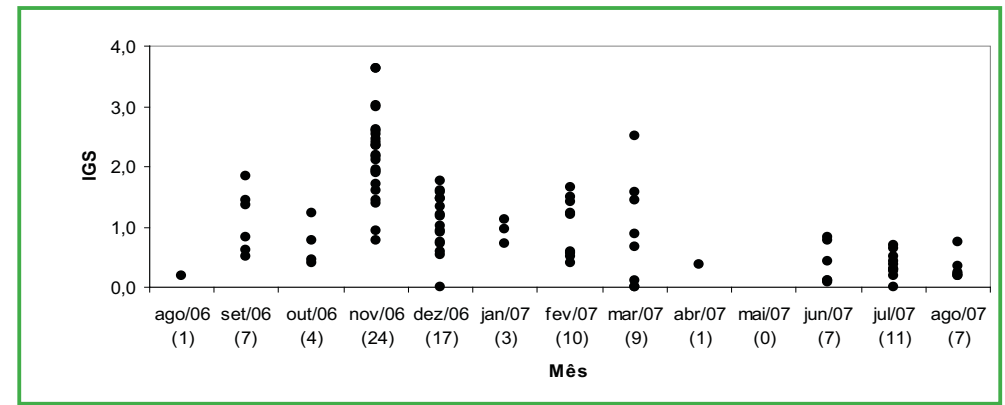

FIGURA 5: Distribuição mensal dos valores individuais de IGS de machos de Trichiurus lepturus desembarcadas pela pesca de pequena escala no litoral norte de Santa Catarina, entre agosto de 2006 e agosto de 2007. Entre parênteses, o tamanho da amostra. 
Das três artes de pesca analisadas, a de fundeio foi a que apresentou maior freqüência de exemplares em reprodução: $62 \%$ encontravam-se em estádio C, Ch, D ou E (Tabela 2). Apenas em agosto de 2006 e em maio - ausência da espécie no fundeio - não foram registrados indivíduos nessa condição (Figura 6). $\mathrm{Na}$ pesca de caceio a freqüência de exemplares em estádio C, Ch, D ou E foi menor: 44\% (Tabela 2). Entretanto, em todos os meses em que a espécie ocorreu no caceio, indivíduos em reprodução foram registrados (Figura 6). $\mathrm{Na}$ pesca de arrasto não foram registrados indivíduos em atividade reprodutiva (Tabela 2).

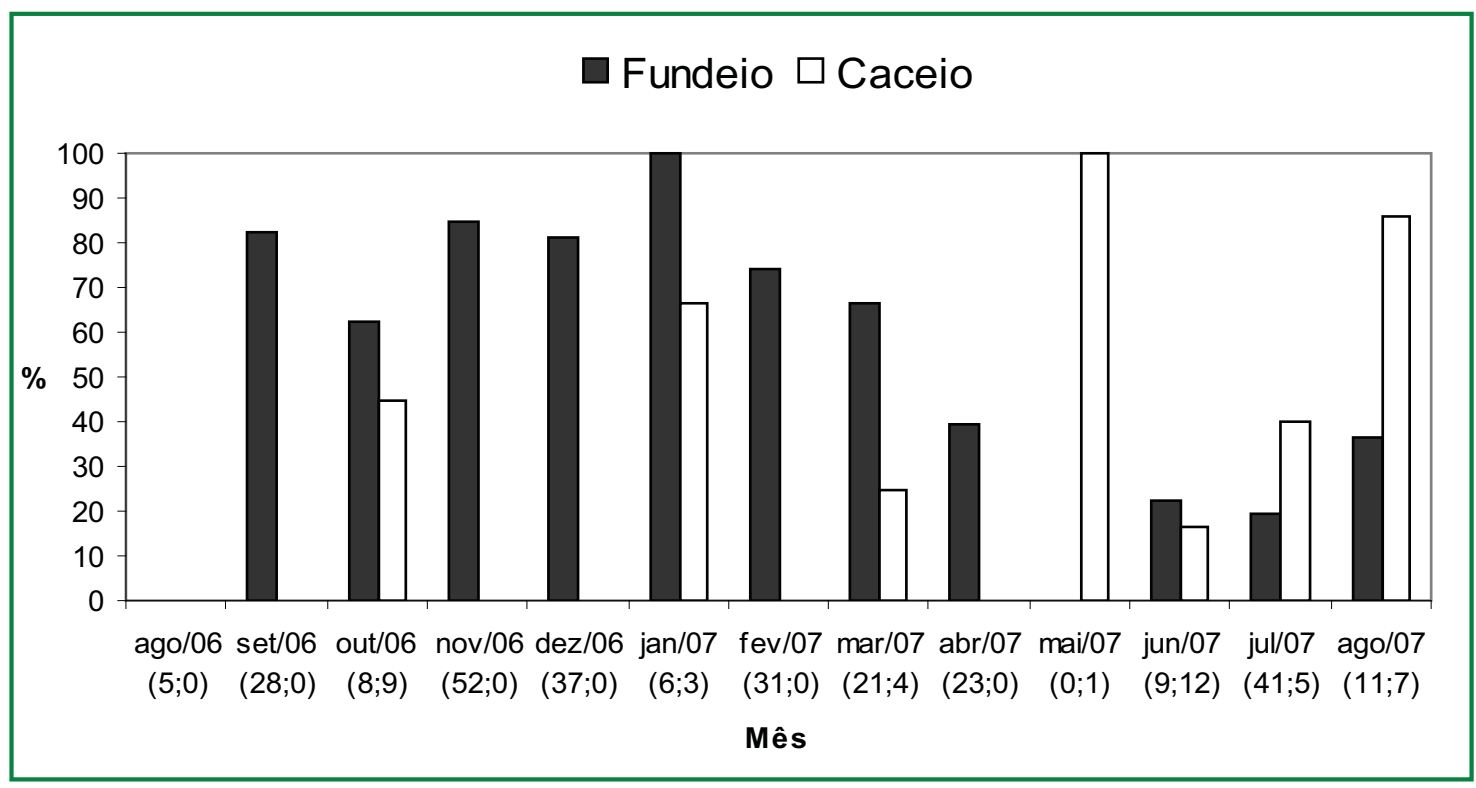

FIGURA 6: Proporção de exemplares de Trichiurus lepturus em reprodução segundo a arte de pesca e o mês, de agosto de 2006 a agosto de 2007. Entre parênteses, o tamanho da amostra em fundeio e caceio, respectivamente. Na pesca de arrasto não foram registrados indivíduos em reprodução.

TABELA 2: Participação relativa (P.R.) das artes de pesca no total de exemplares de Trichiurus lepturus analisados e freqüência de ocorrência dos estádios de maturação (E.M.) registrados em cada uma e no conjunto das três. Todos os valores em \%.

\begin{tabular}{cccccccc}
\hline \multirow{2}{*}{ Artes } & & \multicolumn{7}{c}{ Estádios de maturação } & \multirow{2}{*}{ Total E.M. } \\
\cline { 3 - 7 } & P.R. & A & B & C,Ch & D,E & C,Ch,D,E & \\
\hline \multirow{2}{*}{ Fundeio } & 68,3 & 3,3 & 34,9 & 54,8 & 7,0 & 61,8 & 100,0 \\
Caceio & 10,3 & 2,4 & 53,7 & 24,4 & 19,5 & 43,9 & 100,0 \\
Arrasto & 21,4 & 96,5 & 3,5 & 0,0 & 0,0 & 0,0 & 100,0 \\
\hline Conjunto & - & 23,1 & 30,2 & 39,9 & 6,8 & 46,7 & - \\
\hline
\end{tabular}




\section{Discussão}

A elevada proporção de fêmeas de T. lepturus entre os exemplares de maior porte, também registrada por Ros Pichss e Castillo (1978), Jean e Lee (1984), Munekiyo e Kuwahara (1988), Martins e Haimovici (2000) e Magro (2006), indica que fêmeas atingem tamanhos maiores que machos de mesma idade. Em conseqüência, segundo os dados obtidos, caceio e fundeio, por selecionarem indivíduos maiores que o arrasto, são mais seletivos que este para a captura de fêmeas.

A freqüência temporal de estádios de maturação e os valores individuais de IGS evidenciaram que o período reprodutivo estendeu-se por primavera e verão, entretanto a desova ocorreu ainda no final do outono e do inverno. A atividade reprodutiva de $T$. lepturus por longo período do ano é confirmada por outros trabalhos, seja por análise de ovos (Nakatani et al., 1980), seja por análise da maturação gonadal dos adultos (Magro, 2005). No sul do Brasil, Martins e Haimovici (2000) obtiveram evidências disso na quebra de talude, embora na plataforma a atividade estivesse restrita a primavera e verão. $\mathrm{Na}$ costa Sudeste-Sul, Magro (2005) registrou atividade reprodutiva abrangendo toda a primeira metade do ano, principalmente no verão e no princípio do inverno. Também na Ásia, região de Taiwan, Jean e Lee (1984) estimaram desova ao longo de seis meses, com picos em maio e junho, ou seja, primavera. E no Golfo do México, em profundidades até 91 metros, Sheridan et al. (1984) constataram desova durante seis meses, da primavera ao outono, com pico no verão.

Conclui-se que a atividade pesqueira na região estudada vem atuando sobre exemplares em franca atividade reprodutiva, não apenas pelo tamanho de captura, mas também pela época, visto que na região os desembarques de fundeio (aqueles cujos exemplares são de porte maior) ocorrem majoritariamente na primavera (Robert, 2004).

Os comprimentos médios de primeira maturação obtidos neste trabalho $(75,0 \mathrm{~cm}$ e $64,0 \mathrm{~cm}$, fêmeas e machos respectivamente) diferiram parcialmente daqueles registrados por Magro (2005) - 60,5cm em fêmeas; Magro (2006) - de 65,0 a 67,0cm em fêmeas e de 53,0 a 65,0cm em machos; e por Martins e Haimovici (2000) - 69,3 cm em fêmeas e $63,9 \mathrm{~cm}$ em machos. As diferenças podem ser creditadas a atributos populacionais ou metodológicos, os primeiros envolvendo variáveis temporais e geográficas, e os segundos os métodos empregados para reconhecimento de exemplares jovens e adultos e a própria delimitação das classes de tamanho utilizadas. É certo que na região os jovens de peixe-espada são vulneráveis às três artes estudadas, sobretudo ao arrasto. Igualmente, os adultos são vulneráveis às três, mas em menor grau ao arrasto. O fundeio incide mais sobre indivíduos reprodutivos que o caceio, por ao primeiro serem vulneráveis os indivíduos de maior porte. Todavia, num manejo com preocupação conservacionista, isso não seria motivo para privilegiar-se o caceio sobre o fundeio, pois muitos dos indivíduos de maior porte, mais vulneráveis ao fundeio, são capturados provavelmente em segunda ou terceira maturação gonadal.

Conforme Magro (2006), as taxas de mortalidade total e por pesca apontam que no Sudeste-Sul do Brasil o peixe-espada vem sendo explotado com valores próximos ou acima dos pontos-limite de referência obtidos na análise de rendimento por recruta, e assim a autora alerta para o risco de no Sul-Sudeste estar em curso um processo de sobrepesca. Considerando que na região estudada pelo presente trabalho o arrasto já é interditado durante a primavera, recomenda-se reduzir a intensidade de uso de redes de emalhe - caceio e fundeio - durante a primavera e principalmente o verão. Dessa forma, estará aliviada em seus momentos mais críticos a pressão por pesca de recrutas e de adultos reprodutivos, reservando-se outono e inverno para, com uso de fundeio e caceio, a captura dos indivíduos adultos que estão em menor grau de atividade reprodutiva.

\section{Agradecimentos}

Ao conselho Nacional de Desenvolvimento Científico e Tecnológico (CNPq) e aos pescadores da comunidade de pesca de Barra do Saí, Itapoá (SC). 


\section{Referências}

Chaves, P. T.; Robert, M. C. 2003. Embarcações, artes e procedimentos da pesca artesanal no litoral sul do Estado do Paraná, Brasil. Atlântica, Rio Grande, 25 (1): 53-59.

Jean, C. T.; Lee, S. C. 1984. Reproductive biology of the ribbonfishes, Trichiurus lepturus and Trichiurus japonicus, of Taiwan. Bulletin of the Institute of Zoology Academia Sinica, 23 (1): 9-20.

Jocic-Jakubi, B.; Jovic, N. J. 2006. Verbal memory impairment in children with focal epilepsy. Epilepsy and Behaviour, 9: 432439.

Kwok, K. Y.; Ni, I. H. 1999. Reproduction of cutlassfishes Trichiurus spp. from the South China Sea. Marine Ecology Progress Series, 176: 39-47.

Magro, M. 2005. Trichiurus lepturus Linnaeus, 1758. In: Cergole, M. C.; Ávila-da-Silva, A. O. \& Rossi-Wongtschowski, C. L. (Orgs). Análise das principais pescarias comerciais da Região Sudeste-Sul do Brasil: Dinâmica populacional das espécies em explotação. Série Documentos Revizee - Score Sul. Editora da Universidade de São Paulo, São Paulo, Brasil, p.162-66.

Magro, M. 2006. Aspectos da pesca e dinâmica de populações do espada, Trichiurus lepturus (Trichiuridae, Teleostei), da Costa Sudeste-Sul do Brasil. Tese de Doutorado, Universidade de São Paulo, Brasil, 220pp.

Martins, A. S.; Haimovici, M. 2000. Reproduction of cutlassfish Trichiurus lepturus in the southern Brazil subtropical convergence ecosystem. Scientia Marina, 64 (1): 97-105.

Munekiyo, M.; Kuwahara, A. 1984a. Spawning season and sex ratio of ribbon fish (Trichiurus lepturus) in Western Wakasa Bay (Japan). Bulletin of the Japanese Society of Scientific Fisheries, 50 (8): 1279-1284.

Munekiyo, M.; Kuwahara, A. 1984b. Spawning ground, mating systems and distribution pattern of ribbon fish (Trichiurus lepturus). Bulletin of the Japanese Society of Scientific Fisheries, 50 (9): 1527-1534.

Munekiyo, M.; Kuwahara, A. 1988. Age and growth of Ribbon Fish in the Western Wakasa Bay. Bulletin of the Japanese Society of Scientific Fisheries, 54 (8): 1305-1313.
Nakatani, K.; Matsuura, Y.; Sato, G. 1980. Estudo do ciclo de vida do peixe-espada Trichiurus lepturus. Boletim do Instituto Oceanográfico, 29 (2): 255-259.

Park, C. S.; Kim, Y. S.; Hawang, H. J.; Hong, B. K. 1998. On the spawning and maturity of hairtail, Trichiurus lepturus, in the East China Sea. Bulletin of National Fisheries Research and Development Institute, 54: 19-25.

Robert, M. C. 2004. Análise da pesca artesanal de fundeio realizada pelas comunidades de Brejatuba, litoral sul do Paraná, e de Barra do Saí, litoral norte de Santa Catarina, Brasil. Dissertação de Mestrado, Universidade Federal do Paraná, Brasil, 188pp.

Robert, M. C.; Chaves, P. T. 2006. Dinâmica da atividade pesqueira artesanal em duas comunidades da região litorânea limítrofe Santa Catarina - Paraná, Brasil. Boletim do Instituto de Pesca, 32 (1): 15-23.

Ros Pichss, R. M.; Castillo, M. P. 1978. Contribution to knowledge of the biology of the Atlantic cutlassfish Trichiurus lepturus. Ciencias Serie 8 Investigaciones Marinas (Havana), 37: 1-33.

Sheridan, P. F.; Trimm, D. L.; Baker, B. M. 1984. Reproduction and food habits of seven species of northern Gulf of Mexico fishes. Contributions in Marine Science, 27: 175-204.

Souza, L. M.; Chaves, P. T. 2007. Atividade reprodutiva de peixes (Teleostei) e o defeso da pesca de arrasto no litoral norte de Santa Catarina, Brasil. Revista Brasileira de Zoologia, 24 (4): 11131121.

Sunyé, P. S. 2006. Diagnóstico da pesca no litoral do Estado de Santa Catarina. In: Isaac, V. J.; Martins, A. S.; Haimovici, M. \& Andriguetto, J. M. (Orgs). A pesca marinha e estuarina do Brasil no início do século XXI: Recursos, tecnologias, aspectos socioeconômicos e institucionais. Editora Universitária UFPA, Belém, Brasil, p.141-156.

Vazzoler, A. E. A. de M. 1996. Biologia da reprodução de peixes teleósteos: Teoria e prática. Editora da Universidade Estadual de Maringá, Maringá, Brasil, 169pp.

Yamada, U. 1970. The reproductive characteristics of the ribbon fish, Trichiurus lepturus Linnaeus, in the East China Sea. Bulletin Seikai Regional Fisheries Research Laboratory, 41: 63-81. 